\title{
Familienmigration bei Hochqualifizierten: wie intergenerationale Beziehungen das Einleben prägen
}

\author{
Stefanie Föbker \\ Department of Geography, University of Bonn, Bonn, Germany \\ Correspondence: Stefanie Föbker (foebker@geographie.uni-bonn.de)
}

\begin{abstract}
Received: 23 November 2016 - Revised: 13 November 2017 - Accepted: 15 November 2017
\end{abstract}
- Published: 12 December 2017

\begin{abstract}
Kurzfassung. This paper addresses family migration amongst highly-skilled professionals. Drawing on the concept of linked lives, it analyses how the linked lives of parents and children affect integration processes after international migration. The article is based on qualitative interviews with highly-skilled migrants and their accompanying partners in Germany and Great Britain. The analysis illustrates how parents try to reestablish stability and security in their children's lives after migration. It reveals common concerns. However, some of the parents' strategies are location-specific. The results indicate that the parents' efforts for their children's integration also have an effect on their own integration. Given the importance of children in the integration process, the paper suggests paying more attention to the children's perspective in future migration research.
\end{abstract}

\section{Einleitung}

Etwa drei Viertel der hochqualifizierten Arbeitsmigranten in Deutschland leben in einer Partnerschaft, knapp $40 \%$ leben in einem Haushalt mit Kindern. Dies ergab eine aktuelle Erhebung des Bundesamtes für Migration und Flüchtlinge unter Inhabern der Blauen Karte $\mathrm{EU}^{1}$ (Hanganu und Heß, 2016:339ff.). Die Erkenntnisse stehen in einem deutlichen Kontrast zu der öffentlichen Debatte um hochqualifizierte Migranten, in der sie häufig als flexible Individuen gedacht werden, die attraktiven Arbeitsangeboten folgen, um ihre persönlichen Karrieren voranzutreiben. Die aktuellen Ergebnisse zeigen aber, dass auch bei hochqualifizierten Beschäftigten Familienmigration eine wichtige Rolle spielt.

Die wissenschaftliche Auseinandersetzung mit der Migration von Hochqualifizierten im Familienkontext konzentriert sich insbesondere auf Doppelkarrierepaare (KrauseNicolai, 2005; Gramespacher et al., 2010) sowie die berufliche Integration begleitender Partnerinnen und (seltener) Part-

\footnotetext{
${ }^{1}$ „Die Blaue Karte EU ist ein befristeter Aufenthaltstitel für hochqualifizierte Zuwanderer aus Drittstaaten (... ). In Deutschland wird die Blaue Karte EU an Akademiker erteilt, wenn eine Arbeitsplatzzusage für eine hochqualifizierte Beschäftigung vorliegt, mit der ein bestimmtes Regelmindestgehalt erzielt wird.“ (Hanganu und $\mathrm{He}$, 2016:5).
}

ner (Roos, 2013; Braseby, 2010; Purkayastha, 2005). Ebenso gibt es, insbesondere in der Literatur zum internationalen Personalmanagement, umfangreiche Untersuchungen zur Rolle begleitender Partnerinnen für den Erfolg oder Misserfolg internationaler Entsendungen (Gupta et al., 2012; Kupka und Cathro, 2007; kritisch dazu Fechter, 2012). Während der Begriff der Begleitung auf eine passive Rolle der Partnerinnen verweist, widersprechen die empirischen Erkenntnisse einer solchen Generalisierung und verdeutlichen vielmehr, dass Migrationsentscheidungen innerhalb von Familien aktiv ausgehandelt werden und gemeinsame Anpassungsstrategien im Ankunftskontext entwickelt werden (Roos, 2013:156). Neben den Beziehungen zwischen erwachsenen Haushaltsmitgliedern prägen auch die Bedürfnisse und Aktivitäten von Kindern den Migrationsprozess (Bailey et al., 2004:1619). Ihre Rolle in Haushalten hochqualifizierter Migranten ist noch weitgehend unerforscht (vgl. aber Hatfield, 2010). Hier setzt der vorliegende Beitrag an und untersucht, wie sich die Migration mit Kindern auf das Einleben ${ }^{2}$ und die Integration ihrer Eltern im Ankunftskontext auswirkt.

\footnotetext{
${ }^{2}$ Integration wird als langandauernder Prozess beschrieben (vgl. Esser, 2003). In diesem Beitrag wird zusätzlich der Begriff des Einlebens genutzt, um auf die erste Phase der Integration unmittelbar nach einer Migration zu verweisen.
} 
Hierzu wird im folgenden Abschnitt das theoretische Konzept der linked lives vorgestellt, das auf die Verknüpfung verschiedener Lebensläufe im Migrationsprozess verweist (Coulter et al., 2016). Daran anschließend wird der Integrationsbegriff im Zusammenhang mit der Migration Hochqualifizierter diskutiert. Nach der Beschreibung der empirischen Basis, drei qualitativer Studien in Deutschland und Großbritannien, wird schließlich anhand des empirischen Materials nachvollzogen, wie Eltern das Einleben ihrer Kinder nach einer Migration gestalten und welche Effekte das Engagement der Eltern für ihre Kinder auf die eigene Integration hat.

\section{Lebenslaufkonzept und linked lives}

Seit etwa 20 Jahren prägt der Lebenslaufansatz Studien zu Binnenmigration und Umzugsmobilität (Coulter et al., 2016:356). Ursprünglich von Elder (1974) zur Untersuchung menschlicher Entwicklungsverläufe ausgearbeitet, wurde das Konzept in der Mobilitätsforschung aufgegriffen, da es, besser als das zuvor dominante Modell des Lebenszyklus, in der Lage ist, die Destandardisierung und Diversität individueller Lebensverläufe abzubilden (Coulter et al., 2016:355). Vor dem Hintergrund, dass Menschen parallel unterschiedliche Rollen innehaben, vertritt der Lebenslaufansatz die Idee verschiedener miteinander verknüpfter Karrieren im Lebenslauf (z.B. Wohnkarriere, Bildungskarriere, Berufskarriere, Familienkarriere etc.). Der Lebenslauf ist zudem in einen raumzeitlichen Kontext eingebettet, der eine maßgebliche Rolle für die Entwicklung dieser Karrieren spielt (Elder, 1998:4). In der Mobilitätsforschung wird untersucht, wie Ereignisse in diesen Karrieren mit Migrationsprozessen in Wechselwirkung stehen. So ist beispielsweise bei hochqualifizierten Beschäftigten die Verknüpfung von internationaler Mobilität und beruflichem Aufstieg zu einem Imperativ geworden, etwa innerhalb multinationaler Konzerne (z.B. Millar und Salt, 2008) und in der Wissenschaft (z.B. Bauder et al., 2016).

Ein zentraler Gedanke des Lebenslaufansatzes ist das Prinzip der linked lives, das sich auf die Verknüpfung der Lebensläufe verschiedener Individuen bezieht. Dem linked lives Prinzip zu Folge haben Menschen im Verlauf ihres Lebens soziale Beziehungen mit Verwandten, Freunden etc., die durch ihre Meinungen und Handlungen individuelle Entscheidungen beeinflussen. Ein besonderes Augenmerk liegt auf intergenerationalen Beziehungen. So haben die Erfahrungen und Entscheidungen von Eltern eine große Bedeutung für die Entwicklung ihrer Kinder, ebenso wirkt die Entwicklung der Kinder auf die Lebensumstände der Eltern ein (Elder, 1998:3ff.).

Entsprechend werden Mobilitätsentscheidungen im Kontext von linked lives untersucht. Anstatt einen Umzug als Ereignis zu verstehen, das ein einzelnes Individuum an einem bestimmten Zeitpunkt erlebt, wird er zunehmend als relationale Praxis verstanden, als Bewegung, die Menschen über
Zeit und Raum hinweg verbindet (Findlay et al., 2015:391; Coulter et al., 2016:358). Dabei können Relationen auf unterschiedlichen Ebenen ausgemacht werden. Zum einen bestehen Verknüpfungen zwischen den Personen innerhalb eines mobilen Haushaltes. Dort werden Mobilitätsentscheidungen ausgehandelt zwischen Individuen mit unterschiedlichen Präferenzen und ungleichen Machtpositionen (Findlay et al., 2015:395; Coulter et al., 2016:359). Zum anderen wirken auf Mobilitätsentscheidungen auch Verknüpfungen zu nicht mobilen Personen außerhalb des Haushaltes (z.B. Großeltern, erwachsene Kinder und Kinder in Nachtrennungsfamilien, die im Haushalt des anderen Elternteils leben) (vgl. Mulder, 2007; Feijten und van Ham, 2013:450). Auch sie können Mobilität hervorrufen, ermöglichen oder beschränken, wenn ihnen gegenüber Bindungen, Verpflichtungen oder Unterstützungsbeziehungen bestehen (Bailey et al., 2004:1620). Soziale Netzwerke wirken somit als Ressourcen und als Restriktionen im Mobilitätsprozess; gleichzeitig werden soziale Beziehungen durch Mobilität auch verändert (Coulter et al., 2016:353).

Untersuchungen, die sich mit den Verknüpfungen von Kindern und Eltern im Kontext internationaler Wanderungen befassen, zeigen, dass die Präsenz von Kindern im Haushalt die Wanderungsentscheidung positiv beeinflussen kann, wenn mit der Migration verbesserte Zukunftsperspektiven für die Kinder, insbesondere hinsichtlich Bildung, Versorgung und Sicherheit verbunden werden (Müller-Bachmann, 2014:116; Salazar-Parreñas, 2005). Gerade die Einbindung in das Bildungssystem ist aber auch eine zentrale Hürde, wenn aus der Migration eine Unterbrechung der Schullaufbahn resultiert. Soziale Netzwerke der Kinder und Netzwerke zur Kinderbetreuung sind weitere Argumente, die gegen Migration oder für eine Migration der Eltern ohne ihre Kinder angeführt werden (Jurt und Roulin, 2015:135; Pusch, 2010:297). Spielen die Bedürfnisse von Kindern als Argument in der Migrationsentscheidung sowie hinsichtlich Zeitpunkt und Ziel (Ryan und Sales, 2013) eine wesentliche Rolle, so hat sich ihr aktiver Einfluss im tatsächlichen Entscheidungsprozess häufig als gering erwiesen. Kinder werden in unterschiedlichem Maße an der Entscheidungsfindung beteiligt (Bushin, 2009:432), allerdings werden die Migrationsentscheidungen meist von Erwachsenen getroffen (Skelton, 2009:1441).

Sowohl bei der Bewertung von Migration als auch bei der Einbindung von Kindern in Entscheidungsprozesse spielen gesellschaftliche Vorstellungen über Kindheit und Elternschaft eine wichtige Rolle. Im Hinblick auf westliche Gesellschaften dominieren dabei zwei Argumentationslinien. Zum einen wird Kindheit als eine Zeit der Freiheit und Sorglosigkeit konzipiert, die nicht durch Verantwortungen beeinträchtigt wird (Bushin, 2009:438). Zum anderen werden Kinder als vulnerabel verstanden. Die Aufgabe der Familie ist demnach, ihnen Sicherheit und Stabilität zu bieten (Hutchins, 2011:1232; NiLaoire et al., 2010:156). Je nach dem Herkunfts- und Zielkontext kann mit dem Wechsel des 
Wohnstandortes ein Gewinn an Sicherheit und Stabilität verbunden sein oder kann Migration an sich als komplexer Prozess verstanden werden, der mit Unsicherheiten und Instabilität verbunden ist (Hutchins, 2011:1227, 1232; Bushin, 2009:438). In diesem Zusammenhang verweist der linked lives Ansatz darauf, dass elterliches Handeln negative Migrationsfolgen, wie den Verlust sozialer Netzwerke, abfedern kann (Hagan et al., 1996:382). Die Verknüpfung von Eltern und Kindern ist somit auch im Integrationsprozess bedeutsam.

\section{Integration hochqualifizierter Migranten}

Integration ist ein zentrales Thema in der gesellschaftspolitischen und wissenschaftlichen Debatte um Zuwanderung. In Deutschland ist der Integrationsbegriff geprägt durch die Arbeiten Hartmut Essers (2003), die kontrovers diskutiert werden (z.B. Pries, 2003). Esser untergliedert Integration in vier Dimensionen: Kulturation (z.B. Sprachkenntnisse, Normenkenntnis), Platzierung (z.B. Arbeitsmarktposition, Position im Wohnungsmarkt), Interaktion (Netzwerke, Partizipation) und Identifikation (Zugehörigkeitsgefühl). Differenziert nach diesen analytischen Dimensionen betrachtet Esser, in welche bestehenden sozialen Systeme, z.B. ethnische Gruppe oder Ankunftsgesellschaft, sich Migranten integrieren (Esser, 2003:7). In der wissenschaftlichen Debatte wird unter anderem kritisiert, dass das klassische Integrationsverständnis vor allem bei den Migranten ansetzt und dabei die Rolle der Aufnahmegesellschaft vernachlässigt (ARL, 2016:2). Auch ist mit Integration häufig die Vorstellung ,relativ einheitlicher sozial-kultureller Identifikations- und geographisch-territorialer Integrationsräume" (Pries, 2003:22) verbunden, in die sich Zuwanderer eingliedern sollen. Sie lässt außer Acht, dass ein Aufnahmekontext weder homogen noch statisch ist. Vor dem Hintergrund der Debatte um transnationale Migration kritisiert Pries zudem, dass Integration zumeist ausschließlich im Ankunftskontext betrachtet und kaum berücksichtigt wird, dass Menschen gleichzeitig in unterschiedlichen lokalen, regionalen und nationalen Kontexten integriert sein können (Pries, 2003:30).

In der Debatte um Integration wurden hochqualifizierte Migranten lange Zeit kaum thematisiert (Kolb, 2006:169). Zum einen wurde angenommen, dass sie strukturell über den Arbeitsplatz integriert sind und aufgrund hoher Einkommen auch keine Probleme beim Zugang zum Wohnungsmarkt bestehen. Zum anderen spielten Fragen des Spracherwerbs, sozialer Kontakte oder gar eines Zugehörigkeitsgefühls zu dem Land, in dem sie leben, bei hochqualifizierten ausländischen Beschäftigten keine Rolle. Denn man ging davon aus, dass sie sich nur für einen befristeten Zeitraum im Ausland aufhalten und aufgrund der großen zeitlichen Beanspruchung durch den Beruf keine Gelegenheit, aber auch keine Notwendigkeit einer über den Arbeitsplatz hinausgehenden Inte- gration bestehen. Die Gruppe der hochqualifizierten Migranten erweist sich bei genauerer Betrachtung jedoch als äußerst heterogen (Föbker et al., 2016:120f.), und es wird deutlich, dass sich die Annahmen zu ihrer Integration am Bild eines flexiblen Individuums orientieren. Sie berücksichtigen nicht, dass viele Wanderungen im familiären Kontext erfolgen und in vielen Fällen die Haushalte hochqualifizierter Migranten aus mehreren Personen bestehen, die das Einleben gegenseitig beeinflussen.

Der Ansatz der linked lives kann sich daher auch für die Untersuchung der Integration von hochqualifizierten Migranten als fruchtbar erweisen. Ihre sozialen Beziehungen stellen Ressourcen und Restriktionen für die Integration dar. Im Folgenden sollen anhand qualitativer Interviews die linked lives von Eltern und Kindern untersucht werden. Dabei konzentriert sich der Beitrag auf einen Ausschnitt dieser Beziehung, nämlich darauf, was Eltern für die Integration ihrer Kinder unternehmen und wie dieses Handeln schließlich auf ihre eigene Integration zurückwirkt. Um die Kritik am klassischen Integrationskonzept aufzugreifen, sollen dabei auch die Rolle der Aufnahmegesellschaft und die Heterogenität des Ankunftskontextes berücksichtigt werden. Das Konzept Essers ist insofern nützlich, als die vier analytischen Dimensionen von Integration (Kulturation, Platzierung, Interaktion und Identifikation) eine differenzierte Betrachtung des mitunter gegenläufigen Einflusses sozialer Beziehungen auf Integration erlauben.

\section{Empirische Basis}

Basis der folgenden Auswertung sind drei Projekte zur Integration hochqualifizierter ausländischer Beschäftigter ${ }^{3}$ und ihrer Familien in Deutschland und Großbritannien. Die Projekte bauen inhaltlich aufeinander auf, waren jedoch nicht als internationale Vergleichsstudie angelegt. In den beiden Projekten in Deutschland (2009-2014) standen hochqualifizierte Migrantinnen und Migranten im Mittelpunkt. Ergänzend wurde eine kleine Zahl von Interviews mit Familienmigrantinnen geführt. Darunter werden hier Personen verstanden, die nicht einem Arbeitsangebot, sondern einem hochqualifizierten Arbeitsmigranten/einer hochqualifizierten Arbeitsmigrantin gefolgt sind. Darauf aufbauend wurde ein Projekt entwickelt, das sich explizit Familienmigranten und -migrantinnen widmet und 2013 in Großbritannien durchgeführt wurde.

Ein Vergleich der Interviews aus Deutschland und Großbritannien bietet die Möglichkeit, allgemeine Strategien und ortsspezifische Besonderheiten zu identifizieren und somit die Bedeutung von unterschiedlichen räumlichen Kontexten für den Einlebensprozess zu verstehen. Dabei ist sowohl die Makroebene der Nationalstaaten als auch die Mesoebene des

\footnotetext{
${ }^{3}$ Bei der Auswahl der Arbeitsmigranten war leitend, dass sie über einen Universitätsabschluss verfügen und ein Arbeitsangebot im Ausland angenommen haben.
} 
lokalen und regionalen Kontextes von Interesse. Die Studien in Deutschland wurden in nordrhein-westfälischen Städten, die Studie in Großbritannien in Städten in den East Midlands durchgeführt. In beiden Regionen sind die Städte durch $\mathrm{Zu}$ wanderung geprägt, sie sind jedoch keine Global Cities. Die Studien haben damit eine neue Perspektive auf die Zuwanderung von Hochqualifizierten, die in der Vergangenheit meist in Global Cities untersucht wurde.

In allen Projekten wurden leitfadengestützte Interviews geführt, die den Einlebensprozess, die Arbeitsmarktintegration und soziale Netzwerke behandeln. Um eine Diversität bei den Gesprächspartnern zu erreichen, wurde für ihre Auswahl ein Set von Merkmalen zugrunde gelegt, das u.a. Geschlecht, Nationalität und Beruf/berufliche Position umfasst. Es wird angenommen, dass diese Merkmale den Verlauf des Eingliederungsprozesses beeinflussen. Der Kontakt zu den Interviewpartnern wurde über den Arbeitsplatz, soziale Netzwerke, internationale Schulen und im Schneeballverfahren hergestellt. Die Gespräche wurden in Englisch oder Deutsch geführt, aufgezeichnet und transkribiert.

Für den vorliegenden Aufsatz wurden aus den drei Projekten die 45 Fälle ausgewertet, in denen die Gesprächspartner mit ihren Kindern im Ausland lebten (vgl. Tabelle 1). In Deutschland wurden 15 Hochqualifizierte, darunter sieben Frauen und acht Männer sowie acht Familienmigrantinnen befragt. Das Sample in Großbritannien setzt sich zusammen aus 20 Frauen und zwei Männern, die Hochqualifizierte auf ihrer Migration begleitet haben. Die Rekrutierung männlicher begleitender Partner erwies sich als äußerst schwierig. Es gab eine deutlich geringere Teilnahmebereitschaft unter potenziellen männlichen Interviewpartnern ${ }^{4}$. Die Gründe dafür bleiben offen, da die angefragten männlichen Familienmigranten nicht begründet haben, warum sie nicht für ein Gespräch zur Verfügung standen. Zudem stellen Männer insgesamt eine Minderheit unter den Familienmigranten dar (vgl. Raghuram, 2004:308; Ackers, 2004:192; Roos, 2013:148). Die Gründe dafür sind vielfältig. So sind gesellschaftliche Normen von Bedeutung, nach denen Männer in Partnerschaften häufig älter und damit auch in der Karriere weiter fortgeschritten sind (Ackers, 2004:198). Außerdem stellt Linehan (2002:808) in einer Studie zu international mobilen Managerinnen fest, dass mangelnde berufliche Optionen für den Partner einen wichtigen Hinderungsgrund für die internationale berufliche Mobilität von Frauen darstellen und sich somit gesellschaftliche Rollenerwartungen auf Migrationsentscheidungen innerhalb von Partnerschaften auswirken. Schließlich verweist Raghuram (2004:305) auch auf den strukturellen Einfluss internationaler Arbeitsmärkte, in denen Berufe, die in der Mehrzahl Frauen ausüben, keine Entlohnung bie-

\footnotetext{
${ }^{4} \mathrm{Da}$ in den Projekten in Deutschland der Schwerpunkt auf den Gesprächen mit Hochqualifizierten lag, wurde die Rekrutierung männlicher Partner nicht weiter forciert. Im Projekt in Großbritannien gab es einen weiteren begleitenden Partner, der hier nicht berücksichtigt wird, da er kein Kind hat.
}

ten, die eine internationale Migration von Familienmitgliedern erlaubt.

Tabelle 1 zeigt weiter, dass fast alle Familienmigrantinnen und -migranten ebenfalls über einen Hochschulabschluss verfügen. Die Herkunftsländer verteilen sich über fast alle Erdteile, wobei jedoch ein deutlicher Schwerpunkt auf Europa liegt. Dies entspricht dem tatsächlichen Wanderungsgeschehen, in dem die Mehrheit der Zuwanderer aus benachbarten Ländern stammt (Eurostat, 2016). Die Struktur des Samples ist bei der Interpretation der Ergebnisse zu berücksichtigen.

\section{Ergebnisse: Eltern gestalten das Einleben ihrer Kinder}

Im Folgenden wird zunächst dargestellt, wie Eltern die Einlebensphase ihrer Kinder nach einer internationalen Migration begleiten (Abschnitt 5.1). Daran anschließend werden zwei Aspekte der Eingliederung der Kinder betrachtet, die von den Eltern als zentral gekennzeichnet werden: der Erwerb der Landessprache (Abschnitt 5.2) und die Integration in das Schulsystem (Abschnitt 5.3). Es wird jeweils beschrieben, was Eltern unternehmen, um das Einleben ihrer Kinder möglichst reibungslos zu gestalten. Daran anschlieBend werden die Effekte des Handelns der Eltern auf ihre eigene Integration untersucht. Dabei wird die Einbettung in den jeweils spezifischen räumlichen Kontext berücksichtigt, indem Unterschiede zwischen dem Sample aus Deutschland und Großbritannien identifiziert werden.

\subsection{Einlebens-Arbeit statt Erwerbsarbeit}

Internationale Mobilität ist ein wichtiger Aspekt für die berufliche Entwicklung von Hochqualifizierten. Im Hinblick auf Kinder wird Migration hingegen häufig mit negativen Erwartungen, wie dem Verlust von Freunden und Nachteilen für die Schullaufbahn, assoziiert (Sime und Fox, 2015). Diese Bewertung hat Folgen für das Handeln der Eltern nach der Migration. So berichten viele Gesprächspartner, dass ein Elternteil sich nach der Migration zunächst dem Einleben der Kinder widmet.

Ich habe mir selbst und [Name des Ehemannes] versprochen, dass mein Fokus in den ersten vier, fünf Monaten die Kinder sind und es darum geht, dass sie sich gut in der Schule einleben. (Familienmigrantin aus Dänemark, in Großbritannien*)

Es sind insbesondere die begleitenden Partnerinnen und Partner, die ihre Kinder in der Einlebensphase praktisch und emotional unterstützen (Purkayastha, 2005:183). Für die hochqualifizierten Arbeitsmigrantinnen und -migranten stehen häufig die Anforderungen der neuen Arbeitsstelle im Vordergrund. So erläutert Jennifer ${ }^{5}$, die einen Posten im Ma-

\footnotetext{
${ }^{5}$ Alle Namen sind Pseudonyme. Aus dem Englischen übersetzte Zitate sind mit* gekennzeichnet.
} 
Tabelle 1. Samplestruktur.

\begin{tabular}{|c|c|c|c|c|c|c|}
\hline \multirow[t]{2}{*}{ Sample } & \multicolumn{5}{|c|}{ Migrationszweck } & \multirow[t]{2}{*}{ Herkunftsländer } \\
\hline & & gesamt & $\begin{array}{l}\text { davon } \\
\text { weiblich }\end{array}$ & $\begin{array}{l}\text { davon } \\
\text { männlich }\end{array}$ & $\begin{array}{l}\text { davon mit } \\
\text { Universitäts- } \\
\text { abschluss }\end{array}$ & \\
\hline \multirow[t]{2}{*}{ Deutschland } & Familie & 8 & 8 & 0 & 8 & $\begin{array}{l}\text { Belgien, Frankreich, Großbritannien, } \\
\text { Niederlande, Österreich, Portugal, Indien, } \\
\text { USA }\end{array}$ \\
\hline & Arbeit & 15 & 7 & 8 & 15 & $\begin{array}{l}\text { Großbritannien, Irland, Niederlande, Portu- } \\
\text { gal, Schweiz, Spanien, Tschechien, Weiß- } \\
\text { russland, China, Indien, Japan, Taiwan }\end{array}$ \\
\hline \multirow[t]{2}{*}{ Großbritannien } & Familie & 22 & 20 & 2 & 20 & $\begin{array}{l}\text { Dänemark, Deutschland, Schweiz, Spanien, } \\
\text { Malawi, Nigeria, Indien, Russland, Chile, } \\
\text { Jamaica, Mexiko, USA }\end{array}$ \\
\hline & Arbeit & nicht $r$ & $r t$ & & & \\
\hline
\end{tabular}

Quelle: eigene Erhebung.

nagement eines internationalen Konzerns in Deutschland angenommen hat, wie sie zu Beginn ihrer Tätigkeit direkt nach der Migration ein neues Team von Kollegen aufbauen musste. Sie ist der Auffassung, dass ihre Karriere und ihre berufliche Mobilität ohne ihren Partner nicht möglich wären.

Als meine Tochter geboren ist (...), hat mein Mann sich entschlossen, nicht mehr zu arbeiten (... ) Er war die ganze Zeit Vollzeitvater. Und das ermöglicht uns ins Ausland zu ziehen. (Hochqualifizierte aus Irland, in Deutschland*)

Dass hier das Beispiel eines männlichen Partners angeführt wird, soll nicht darüber hinwegtäuschen, dass es sich in der Mehrzahl - aber eben nicht ausschließlich - um begleitende Partnerinnen handelt. Während Jennifers Aussage eine Kontinuität der Rollen in ihrer Partnerschaft belegt, war für Hanne die internationale Migration ein Anlass die Rollenverteilung innerhalb der Partnerschaft zu verändern.

Wir waren gleich in Dänemark, gleiche Bezahlung, gleiche Arbeitszeit und gleiche Pflichten, die Kinder zur Schule zu bringen (...), und ich habe entschieden, dass das hier nicht sein muss. (Familienmigrantin aus Dänemark, in Großbritannien*)

Insbesondere bei zeitlich befristeten Auslandsaufenthalten und in der Anfangsphase werden solche Rollenwechsel bewusst gewählt und nicht nur als Verpflichtung, sondern auch als Möglichkeit gesehen, mehr Zeit mit den Kindern zu verbringen (Raghuram, 2004:307). Das gilt auch für begleitende Väter. So betont Filipe, der nach Großbritannien gezogen ist, weil seine Frau dort eine Promotionsstelle angetreten hat und der dort selber in Teilzeit als Techniker in einer Schule arbeitet:
Ich würde gerne was machen, was mehr mit meinem richtigen Job zu tun hat (...). Aber ich bin sehr froh, insbesondere weil ich mich um meine Tochter kümmern kann, was sehr wichtig für mich ist. Das ist meine Priorität. (Familienmigrant aus Spanien, in Großbritannien*)

Filipes Beispiel verweist auch auf die beruflichen Kompromisse, die viele Partnerinnen und Partner eingehen, wenn sie sich für eine Familienmigration entscheiden. Negativ bewertet wird die Konzentration auf die Familie dann, wenn sie nicht freiwillig erfolgt. Dies ist auch eine Konsequenz struktureller Rahmenbedingungen vor Ort. Da infolge der Migration gewohnte soziale Netzwerke zur Kinderbetreuung nicht mehr zur Verfügung stehen, gewinnen lokal verfügbare Betreuungsangebote an Bedeutung. Hier zeigen sich deutliche internationale Unterschiede. Von den Gesprächspartnerinnen in Deutschland werden grundsätzliche Probleme beschrieben, einen Betreuungsplatz zu finden. Besondere Schwierigkeiten bereiten die Betreuung von Babys unter einem Jahr und der zeitliche Umfang der Betreuung, der es zum Teil nicht erlaubt, eine Berufstätigkeit aufzunehmen (Föbker et al., 2014:274). In Großbritannien sind in erster Linie die Kosten der Betreuung von kleinen, nicht schulpflichtigen Kindern ein Hindernis, externe Betreuungsangebote in Anspruch zu nehmen. Betroffen sind Familien mit geringem Einkommen, zum Beispiel Familien von Doktoranden. Hier zeigen sich auch herkunftsspezifische Unterschiede. Insbesondere Partnerinnen aus dem globalen Süden hatten die Migration mit dem Ziel verbunden, in Großbritannien Geld zu verdienen. Um Kinderbetreuungskosten zu vermeiden, weichen sie häufig in Arbeitsverhältnisse im Niedriglohnsektor aus, die eine flexible Arbeitsgestaltung erlauben. 
Die Beispiele belegen, dass die Anwesenheit von Kindern im Haushalt die Integration von begleitenden Partnern und Partnerinnen in den Arbeitsmarkt oftmals beschränkt. Zum einen wird die internationale Migration mit Kindern als Anlass genommen, nicht oder weniger zu arbeiten. Zum anderen wirken insbesondere kleine Kinder als Restriktion, wenn lokal keine passenden Betreuungsangebote verfügbar sind. Auf solche externen Betreuungsdienstleistungen sind Familien nach einer Migration besonders angewiesen, da soziale Netzwerke im Ankunftskontext in der Regel zunächst nicht vorhanden sind. Die linked lives von Eltern und Kindern stellen somit eine Restriktion für die berufliche Integration eines der Elternteile dar.

\subsection{Sprache als Schlüssel zur Integration}

Die besondere Herausforderung vieler internationaler Wanderungen besteht in der Sprachbarriere. Sind Kinder auch bei Binnenmigration mit dem Verlust sozialer Beziehungen konfrontiert und häufig auch mit dem Wechsel zwischen Bildungssystemen, so kommt bei internationaler Migration die Fremdsprache meist erschwerend hinzu. Sie wirkt sich negativ sowohl auf das Knüpfen neuer Kontakte als auch den Einstieg in den Schulalltag aus.

Der Spracherwerb ist somit eines der zentralen Anliegen der Eltern nach der internationalen Migration. Viele Eltern erwarten zunächst Nachteile für ihre Kinder, da sie sich in eine neue Umgebung einfügen müssen, deren Sprache sie nicht beherrschen. Langfristig bewerten viele Eltern die Möglichkeit, dass ihre Kinder mehrsprachig aufwachsen, als positiven Effekt der internationalen Migration. Insbesondere die Eltern im britischen Sample erwarten, dass ihre Kinder davon im späteren beruflichen Leben profitieren. In erster Linie werden Schulen und Kindertageseinrichtungen als Orte identifiziert, an denen Kinder die neue Sprache erlernen. Die Eltern sehen ihre eigene Aufgabe darin, ihre Kinder im Spracherwerb zu unterstützen. Dazu zählt die Entscheidung, jüngere Kinder in Kindertagesstätten anzumelden oder Spielgruppen mit ihnen zu besuchen. Diese Aktivitäten können sich wiederum positiv auf die soziale Integration der Eltern auswirken. So berichtet Kate, die aus Großbritannien nach Deutschland gezogen ist:

Ich habe die meisten meiner Freunde hier in [Ort] und in Deutschland über den Kindergarten kennengelernt, weil die Mütter zur gleichen Zeit kommen, um ihre Kinder hinzubringen und abzuholen. (Familienmigrantin aus Großbritannien, in Deutschland*)

Kindergärten und Grundschulen sind Orte, an denen Eltern im Alltag sowie bei besonderen Veranstaltungen andere Eltern treffen. Sie tragen zur Entstehung neuer sozialer Netzwerke bei und fördern das lokale Zugehörigkeitsgefühl (Collins und Coleman, 2008:290f.). Das gilt nicht nur für Eltern mit Migrationserfahrung. Da Migration jedoch mit dem
Verlust lokaler sozialer Netzwerke einhergeht, besteht hier ein besonderes Bedürfnis, vor Ort neue soziale Kontakte zu knüpfen (Föbker et al., 2014).

Zu der Unterstützung der Kinder beim Spracherwerb zählt auch die Entscheidung der Eltern, selber an einem Sprachkurs teilzunehmen.
Nun wird mein Sohn in den Kindergarten ge- hen. Und jetzt ist die größte Herausforderung: Du kannst nicht Vater und Mutter haben, die nicht [deutsch] lesen und schreiben können. Das ist das schlimmste. Gebildet sein und nicht lesen und schreiben können. Deshalb machen wir jetzt einen Integrationskurs. (Hochqualifizierter aus Indien, in Deutschland*)

Chapal, der zum Zeitpunkt des Gespräches seit gut zwei Jahren als Wissenschaftler in Deutschland arbeitet, hat bisher nicht Deutsch gelernt. Durch seine starke berufliche Einbindung hat er weder Zeit dafür aufbringen mögen, noch hat er eine Notwendigkeit gesehen, da er in einem englischsprachigen Umfeld tätig ist. Erst mit dem Eintritt seines Kindes in das deutsche Bildungssystem verändert sich seine Beurteilung der Situation. Hier zeigt sich deutlich ein Effekt der linked lives verschiedener Generationen innerhalb eines Haushaltes auf die sprachliche Integration seiner Mitglieder.

Der Gesprächspartner wirkt einer Situation entgegen, in der er über geringere Sprachkenntnisse als sein Kind verfügt und es daher im Alltag nicht mehr unterstützen kann. Eine solche dissonante Akkulturation der Generationen einer Familie wird häufig nach der Migration beobachtet (Portes und Rumbaut, 2001:44), wenn Kinder schneller als ihre Eltern die neue Sprache erlernen und die Eltern die Hilfe ihrer Kinder, etwa als Übersetzer, in Anspruch nehmen müssen ( $\mathrm{Si}$ me und Fox, 2015:531). Ein derartiger Rollentausch wurde im untersuchten Sample nicht beobachtet, was nicht zuletzt auf das große kulturelle und soziale Kapital der Gesprächspartner zurückgeführt werden kann. Sie haben entweder selber entsprechende Sprachkenntnisse oder verfügen im beruflichen Kontext über soziale Kontakte zu Muttersprachlern, die als Mittler fungieren können.

Der Fall von Chapal verdeutlicht auch einen Unterschied zwischen den beiden Samples, der auf den Stellenwert der deutschen Sprache im Vergleich zur globalen Lingua franca Englisch zurückzuführen ist. So verfügen im britischen Sample alle erwachsenen Familienangehörigen zumindest über grundlegende Sprachkenntnisse und haben darüber hinaus ein Interesse daran, ihre Sprachkenntnisse weiter zu verbessern, da sie einen persönlichen Nutzen davon erwarteten.

\subsection{Eingliederung in das Schulsystem}

Die Schulwahl ist, unabhängig von der Migrationserfahrung, ein zentrales Thema für Eltern. Insbesondere in Mittelschichthaushalten gelten eine hochwertige Schulbildung und eine erfolgreiche Schullaufbahn als wichtige Voraussetzun- 
gen für eine positive Zukunftsperspektive der Kinder (Holloway et al., 2010:585; Collins und Coleman, 2008:293). Nach einer internationalen Migration befürchten Eltern oftmals, dass es den Kindern schwer fällt, sich aufgrund der Sprachbarriere und des anderen Curriculums in das Bildungssystem zu integrieren. Die Migrationsentscheidung, die die Eltern zugunsten der beruflichen Karriere eines Elternteils getroffen haben, kann sich dann nachteilig auf die Bildungskarriere und damit die Zukunft ihrer Kinder auswirken. Daher ist es den Eltern ein besonders wichtiges Anliegen, eine geeignete Schule zu finden, die den Kindern einen guten Einstieg in die neue Lernumgebung ermöglicht. Diese Sorge teilen Eltern in beiden Samples. Es werden jedoch unterschiedliche Strategien angewandt, um dieser Herausforderung zu begegnen.

Bei den Gesprächspartnern in Deutschland bestehen zum Teil Vorbehalte gegenüber dem Besuch einer deutschen Schule. Der Erwerb der Fremdsprache wird zwar grundsätzlich positiv bewertet, der Unterricht in deutscher Sprache wird jedoch als nachteilig für den Schulerfolg wahrgenommen und die Integration in das deutsche Schulsystem als Hindernis für zukünftige Migration.

Da haben wir gesagt vom niederländischen Schulsystem in das deutsche Schulsystem und dann nach weiß ich wie viel Jahren vielleicht in ein anderes Land umziehen, wieder ein anderes Schulsystem. Das geht nicht für die Kinder. Also haben wir gesagt, dann gehen die hier in die Internationale Schule und dann sind wir mobiler und dann können wir einfacher wechseln. (Familienmigrantin aus den Niederlanden, in Deutschland)

Ein Teil der Familien entscheidet sich für internationale oder bilinguale Schulen, weil sie keine oder nur geringe Deutschkenntnisse verlangen, was sowohl für die Kinder als auch die Eltern den Einstieg in den Schulalltag erleichtert. Sind weitere internationale Umzüge geplant, ist außerdem ein vergleichsweise reibungsloser Wechsel zwischen internationalen Schulen möglich. Jedoch kommen internationale Schulen aufgrund ihrer strukturellen Rahmenbedingungen nicht für alle Eltern in Frage. Zum Ersten sind internationale oder fremdsprachige Schulen in der Mehrzahl Privatschulen, deren Besuch mit hohen Schulgebühren verbunden ist. Zum Zweiten besteht die Zielgruppe dieser Schulen aus Kindern international hochmobiler Eltern und der Anteil deutscher Schüler wird zum Teil begrenzt (Weiss et al., 2014:174). Somit ist diese Schulform unattraktiv für Eltern, die Anschluss an die lokale deutsche Gesellschaft suchen. Zum Dritten sind internationale Schulen vor allem in urbanen Regionen mit einem gewissen Internationalisierungsgrad, wie im Untersuchungsgebiet, zu finden (Weiss et al., 2014:168). In anderen Regionen ist diese Schulform nur eingeschränkt vorhanden.

Tatsächlich hat die Schulwahl einen großen Einfluss auf das Einleben der Familien am neuen Wohnort. So bieten internationale Schulen den Eltern informelle Treffpunkte, was bei weiterführenden deutschen Schulen kaum der Fall ist.
Sie vermitteln aktiv Kontakte zwischen Eltern, um das Einleben in die neue Umgebung zu unterstützen (Weiss et al., 2014:176f.). Gleichzeitig sind die Schulen häufig Teil einer „expatriate bubble“ (Braseby, 2010:127), in der die internationalen Eltern untereinander Kontakte pflegen und kaum Kontakte in die deutsche Gesellschaft außerhalb der Schule entwickeln. Sie stärken somit weniger das lokale Zugehörigkeitsgefühl als die Zugehörigkeit zu einer internationalen Community.

Eltern, deren Kinder deutsche (Grund-)Schulen besuchen, haben hingegen einen direkten Zugang zu den Alteingesessenen. Bei weiterführenden Schulen kommen die Eltern seltener in Kontakt miteinander, und es wird ein geringeres Interesse gegenüber Neuzugezogenen festgestellt, da schon viele Kontakte zu anderen Eltern bestehen. Insgesamt stellt der deutsche Schulalltag die zugewanderten Eltern häufig vor Herausforderungen. So berichtet eine Mutter von ihren Erfahrungen mit Vertretern des deutschen Bildungssystems:
Es gibt dieses Sprach-Ding. Am Anfang konnte ich null Deutsch. Und ich (...) habe den Eindruck, dass die Leute, die in den Kindergärten und Schu- len und Institutionen arbeiten, gar nicht helfen wol- len. Wir fangen an zu verstehen, dass es durchaus möglich ist, Personen zu finden, die Englisch spre- chen (...), aber unser Gefühl ist, dass Leute gar nicht bereit sind zu helfen und zu sagen, ich spre- che Englisch. (Familienmigrantin aus Portugal, in Deutschland*)

Sie bewertet die Einstellung von Lehrern und Schulleitern in Deutschland als zusätzliche Hürde in der Kommunikation mit ausländischen Eltern. Diese Einschätzung wurde von anderen Gesprächspartnern auch in anderen Kontexten, z.B. Ämtern, geteilt. Die Einstellung in deutschen Institutionen spiegelt die öffentliche Debatte um Integration wider, in der gute Deutschkenntnisse eine zentrale Forderung an die Migranten darstellen (Vogel und Rinke, 2008:275). Somit benötigen Eltern, die ihre Kinder auf nationale Schulen schicken, mehr als grundlegende Deutschkenntnisse, um allgemeine Informationen zu verstehen und um mit Vertretern dieser Institutionen in einen Austausch zu treten und ihre eigenen Interessen sowie die ihrer Kinder zu vertreten. Hier wird deutlich, wie das Handeln der Ankunftsgesellschaft das Einleben der Eltern nach der Migration prägt.

Im britischen Sample gibt es kaum Vorbehalte gegenüber dem Einstieg in das nationale Bildungssystem. Das liegt vor allem daran, dass der Unterricht in englischer Sprache nicht als Nachteil, sondern als Vorteil für die Kinder wahrgenommen wird. Als zentrale Herausforderung stellt sich in Großbritannien die Aufnahme in eine gut bewertete Schule heraus, was jedoch nicht spezifisch für Eltern mit $\mathrm{Zu}$ wanderungsgeschichte ist, sondern alle Eltern schulpflichtiger Kinder betrifft. Informationen über die Qualität von Schulen sind öffentlich verfügbar (vgl. https://www.gov.uk/ government/organisations/ofsted). Da die Distanz zwischen 
Wohnort und Schule ein entscheidender Faktor beim $\mathrm{Zu}$ gang zu stark nachgefragten Schulen ist, bemühen sich Eltern schulpflichtiger Kinder um einen Wohnstandort im Einzugsgebiet einer gut bewerteten Schule, auch wenn die Immobilienpreise in diesen Gebieten signifikant höher liegen (Benson et al., 2015:38). Vor dem Hintergrund der Struktur des britischen Wohnungsmarktes, der als Eigentumsmarkt gekennzeichnet werden kann (ONS, 2013), ist der Immobilienerwerb im Einzugsgebiet guter Schulen eine Schlüsselstrategie von Mittelschichteltern, um einen Platz in einer solchen Schule zu ergattern (Smith, 2011:658). Viele Interviewpartner übernehmen diese nationale Strategie, insbesondere dann, wenn sie eine unbefristete Aufenthaltsperspektive haben oder aus Herkunftskontexten stammen, in denen Wohneigentum dominiert.

Es ist also sein [Sohn] zweites Jahr hier an der höheren Schule; und so haben wir das Haus gekauft, nur wegen ihm sozusagen, dass er an diese gute Schule kommt, ansonsten hätten wir ein billiges Haus gekauft, und er wär an ne Privatschule gegangen. (Familienmigrantin aus Deutschland, in Großbritannien)

Als Alternative wird die Wahl einer Privatschule identifiziert. Eltern stehen somit vor der Wahl, ökonomisches Kapital, sofern verfügbar, entweder in den Wohnungsmarkt zu investieren, um Zugang zu einer guten öffentlichen Schule zu erhalten, oder in die Gebühren für Privatschulen (Benson et al., 2015:36). Für Familien, die sich zeitlich befristet in Großbritannien aufhalten und dort kein Eigentum erwerben möchten, stellt sich die Frage, ob und wie sie, ohne Eigentum zu erwerben, einen Wohnstandort im Einzugsgebiet einer guten Schule realisieren können. Teilweise stellen Arbeitgeber Wohnraum zur Miete zur Verfügung, dessen Attraktivität für Familien dann wesentlich von der Zuordnung zum Einzugsgebiet guter Schulen abhängt. Die Präsenz von schulpflichtigen Kindern im Haushalt spielt somit in vielen Fällen eine wichtige Rolle für die Wohnstandortwahl und damit die Frage, in welcher räumlichen und sozialen Umgebung das Einleben in das neue Umfeld stattfindet und zu welchen sozialen Schichten Kontaktmöglichkeiten entstehen.

Die Wahl der Schule kann weitreichende Konsequenzen nicht nur für die Kinder, sondern auch für die begleitenden Partner und Partnerinnen haben, die sich für das Einleben der Kinder engagieren. So schildert eine Mutter, dass sie ihre Tochter auf einer Schule angemeldet hatten, die sich, wie sie später feststellten, durch Disziplinprobleme, abweichendes Verhalten der Schüler und ein mangelndes Vertrauen des Lehrpersonals in die Schüler auszeichnete. Diese Situation hat sich negativ auf das eigene Kind ausgewirkt und auch die Lebensumstände der Eltern in den ersten zwei Jahren des Aufenthalts in Großbritannien geprägt (vgl. Elder, 1998:3ff.). Für die Mutter folgte daraus auch eine Verzögerung der eigenen Arbeitsmarktintegration.
Insgesamt wird deutlich, dass sich die Sorgen der Eltern hinsichtlich des erfolgreichen Einlebens in das Bildungssystem zwischen den beiden Samples unterscheiden. In Deutschland sind sie eng mit der Migration und der Unterrichtssprache Deutsch verknüpft. In Großbritannien hingegen steht die Wahl einer "guten Schule“ im Mittelpunkt. Entsprechend unterscheiden sich die Strategien der Eltern, eine geeignete Schule zu finden. Jedoch spielt in beiden Fällen das ökonomische Kapital der Eltern eine wichtige Rolle für die Umsetzung ihrer Strategien. Die Schulwahl hat schließlich einen wichtigen Einfluss auf den Einlebensprozess des gesamten Haushaltes. Sie beeinflusst, wie viel Zeit der Prozess in Anspruch nimmt, welche Anforderungen er an die Eltern stellt und zu welchen Teilen der Bevölkerung die Familie einen Zugang erhält. Somit wird auch im Zusammenhang mit der Schulwahl ein Effekt der linked lives von Eltern und Kindern im Integrationsprozess deutlich.

\section{Schlussfolgerungen}

Die Ergebnisse zeigen, wie sich Eltern nach einer internationalen Migration für das Einleben ihrer Kinder engagieren. Insbesondere begleitende Partnerinnen und Partner widmen ihren Kindern Zeit, um nach der Migration wieder Stabilität und Sicherheit in deren Leben herzustellen. Die Bemühungen der Eltern, den Spracherwerb ihrer Kinder und die Integration in das Bildungssystem zu unterstützen, wirken sich auch auf ihre eigene Integration aus. Jedoch unterscheiden sich die Effekte in den verschiedenen Dimensionen der Integration.

So kann das Zusammenleben mit schulpflichtigen Kindern die Eltern zum Erwerb der Fremdsprache motivieren und damit zur kulturellen Integration (Esser, 2003) beitragen. Auf die berufliche Integration von Familienmigrantinnen und -migranten hingegen wirken sich Kinder im Haushalt nachteilig aus. Das liegt zum einen temporär an den Aufgaben der Einlebensarbeit, die von den Familienmigrantinnen und -migranten übernommen werden. Zum anderen können sich problematische Integrationsverläufe der Kinder in das Bildungssystem und strukturelle Rahmenbedingungen der Kinderbetreuung negativ auf die Arbeitsmarktintegration auswirken. Hier zeigen sich auch räumliche Unterschiede. In Deutschland führt der Mangel an bedarfsgerechter Kinderbetreuung zu verlängerten Einlebensphasen der begleitenden Partnerinnen. In Großbritannien betrifft das vor allem die Partner und Partnerinnen von Hochqualifizierten mit geringem Einkommen, da die Betreuung dort hohe Kosten erzeugt.

Im Hinblick auf die soziale Integration haben hochqualifizierte Migrantinnen und Migranten mit Kindern vielfältige Zugangsmöglichkeiten zu sozialen Netzwerken, während Hochqualifizierte ohne Kinder häufig primär über ihren $\mathrm{Ar}$ beitsplatz sozial am neuen Wohnort integriert sind (Föbker et al., 2014:276, 2016:120). Insbesondere die Schulwahl nimmt 
Einfluss darauf, in welche Teile der Gesellschaft sich der Haushalt nach der Migration integriert. Auch hier werden räumliche Unterschiede deutlich. In Großbritannien zeigt sich die große Bedeutung der Wohnstandortwahl im Einzugsgebiet von positiv bewerteten Schulen, um Zugang zu begehrten Schulplätzen zu erhalten. Daraus folgt die Integration in eine sozialspezifische Nachbarschaft. In Deutschland werden internationale Schulen nachgefragt, die aufgrund ihrer strukturellen Rahmenbedingungen die Integration in eine Expat Bubble mit hohem Sozialstatus begünstigen. Dabei ist jedoch zu bedenken, dass internationale Schulen in Deutschland nicht in allen Regionen in dem Maße zur Verfügung stehen, wie dies in der Untersuchungsregion der Fall ist. Einlebensstrategien unterscheiden sich somit auch regional.

Die Erkenntnis, dass Kinder für das Einleben der Eltern nach der Migration eine wichtige Rolle spielen, unterstreicht die Bedeutung des Ansatzes der linked lives in der Migrationsforschung. Die dargestellten Ergebnisse beruhen auf Gesprächen mit Eltern. Sie vermitteln erste Einblicke in die Rolle intergenerationaler Beziehungen in Integrationsprozessen hochqualifizierter Migranten, über die bisher wenig bekannt ist. Sie geben jedoch keine Auskunft darüber, was für die Kinder selber zentrale Herausforderungen des Einlebens sind und wie sich das Handeln der Eltern auf die Integration der Kinder auswirkt. So ist die Schulwahl ein zentraler Aspekt im Handeln der Eltern für das Einleben ihrer Kinder. Vor dem Hintergrund, dass Schulcurricula eine wichtige Bedeutung für die Entwicklung von Identitäten und Zugehörigkeiten haben (Holloway et al., 2010:587f.), stellt sich die Frage nach den Effekten der Schulwahl auf die identifikative Integration von Kindern nach der Migration. Auch berichteten die Eltern wenig über die soziale Integration ihrer Kinder. Hier wären die Stimmen der Kinder notwendig, um zu erfahren, was sie selber unternehmen, um neue Kontakte zu knüpfen und wie ihre Handlungen wiederum zur sozialen Integration ihrer Eltern beitragen. So betont Jenks (2009:93f.), dass Kinder aktiv an der Konstruktion ihrer eigenen sozialen Beziehungen und der Beziehungen der mit ihnen verflochtenen Personen beteiligt sind. Er plädiert daher dafür, die sozialen Beziehungen der Kinder selber zu betrachten. Um die linked lives von Eltern und Kindern bei der Migration und Integration Hochqualifizierter umfassender zu untersuchen, ist es sinnvoll, zukünftig die Perspektive der Kinder stärker zu berücksichtigen. Sie sind, so die Kerngedanken der new social studies of childhood, als aktive und kompetente Akteure zu verstehen, die eine eigene Perspektive auf die soziale Welt haben (James und James, 2001:35). Hier kann auch an die angelsächsischen children's geographies angeknüpft werden, denen in der deutschsprachigen Geographie bisher wenig Aufmerksamkeit geschenkt wurde (Hoinle und Calba, 2015:3).

Aus den Ergebnissen lassen sich verschiedene Handlungsempfehlungen ableiten. Für Eltern stellt insbesondere der Zugang ihrer Kinder zum Betreuungs- und Bildungssystem eine zentrale Herausforderung in der Einlebensphase dar.
Hier sind Beratungsangebote notwendig, die den gesamten Haushalt hochqualifizierter Migranten berücksichtigen und somit auch kindbezogene Fragen aufgreifen. Solche Angebote stehen hochqualifizierten Arbeitsmigranten in unterschiedlichem Ausmaß zur Verfügung. Sie werden teilweise von den Arbeitgebern organisiert in Form eigener Beratungsstellen (z.B. https://www.uni-bonn.de/einrichtungen/ welcome-center) oder als Element von Relocation Packages. Letztgenannte Angebote stehen vor allem Managern internationaler Firmen zur Verfügung, wobei das Angebot umso umfangreicher ausfällt, je höher die Position des Beschäftigten ist (Föbker et al., 2016:115). Für solche Beschäftigten, die keine Beratung durch den Arbeitgeber erhalten, gibt es in einigen Städten kommunale Willkommensagenturen, die (hochqualifizierte) Zuwanderer auch zu Fragen des Einlebens der Familie und der Kinder beraten (z.B. https: //welcome.dresden.de). Der weitere Ausbau solcher vom Arbeitgeber und der beruflichen Position (weitgehend) unabhängigen Beratungseinrichtungen wäre wünschenswert.

Da der Besuch der gewünschten Betreuungs- und Bildungsangebote in vielen Fällen hohe Kosten erzeugt (für Privatschulen, Eigentumserwerb etc.), ist es darüber hinaus wichtig, den Zugang auch für weniger einkommensstarke Haushalte zu verbessern. Ein Ansatzpunkt im britischen Kontext ist die Bereitstellung von Gästewohnungen für mobile Beschäftigte, die durch ihre Lage im Einzugsgebiet den Zugang zu hochwertigen Schulen erlauben. Im deutschen Kontext könnte der Nachfrage nach internationaler Bildung durch die Förderung von öffentlichen Schulen mit englischer Unterrichtssprache und einem internationalerem Curriculum entsprochen werden.

Nicht zuletzt spielt für die Integration in das nationale Bildungssystem auch der Umgang von Lehrern und Schulleitern mit fremdsprachigen Eltern eine Rolle. Hier geben die Ergebnisse aus dem deutschen Kontext Anlass, die Einstellung und den Umgang mit Nicht-Muttersprachlern in deutschen Behörden grundsätzlich zu überdenken.

Datenverfügbarkeit. Das Interviewmaterial ist nicht öffentlich zugänglich, da den Gesprächspartnern Vertraulichkeit und Anonymität zugesichert wurde. Dazu zählt, dass die Gesprächsinhalte vertraulich behandelt und ausgewertet werden sowie Informationen in wissenschaftlichen Artikeln nur in anonymisierter Form dargestellt werden.

Interessenkonflikt. Die Autorin erklärt, dass kein Interessenkonflikt besteht.

Danksagung. Die beiden Projekte in Deutschland wurden gemeinsam mit Daniela Imani, Josef Nipper, Marius Otto, Carmella Pfaffenbach, Günther Weiss und Claus-C. Wiegandt bearbeitet, denen ich herzlich danken möchte. Ebenso gebührt mein Dank Heike Jöns, der Gastgeberin meines Forschungsaufenthaltes in 
Großbritannien. Danken möchte ich schließlich auch der Deutschen Forschungsgemeinschaft für die Finanzierung der Projekte in Deutschland und dem Deutschen Akademischen Austauschdienst, der das Projekt in Großbritannien im Rahmen eines Postdoc Stipendiums gefördert hat.

Edited by: Benedikt Korf

Reviewed by: two anonymous referees

\section{Literatur}

Ackers, L.: Managing relationships in peripatetic careers: Scientific mobility in the European Union, Women. Stud. Int. Forum, 27, 189-201, 2004.

ARL: Migration und Raumentwicklung, Positionspapier 105, Hannover, 2016

Bailey, A. J., Blake, M. K., und Cooke, T. J.: Migration, care and the linked lives of dual-earner households, Environ. Plann. A, 36, 1617-1632, 2004.

Bauder, H., Hannan, C.-A., und Lujan, O.: International Experience in the Academic Field: Knowledge Production, Symbolic Capital, and Mobility Fetishism, Popul. Space Place, 23, e2040, https://doi.org/10.1002/psp.2040, 2016.

Benson, M., Bridge, G., und Wilson, D.: School Choice in London and Paris - A Comparison of Middle-class Strategies, Soc. Policy Admin., 49, 24-43, 2015.

Braseby, A. M.: Adaptation of Trailing Spouses: Does Gender Matter? Dissertation at Florida International University, FIU Electronic Theses and Dissertations, Paper 153, 2010.

Bushin, N.: Researching Family Migration Decision-Making: A Children-in-Families Approach, Popul. Space Place, 15, 429443, 2009.

Collins, D. und Coleman, T.: Social Geographies of Education. Looking Within, and Beyond, School Boundaries, Geography Compass, 2, 281-299, 2008.

Coulter, R., van Ham, M., und Findlay, A. M.: Rethinking residential mobility: Linking lives through time and space, Prog. Hum. Geog., 40, 352-374, 2016.

Elder, G. H.: Children of the Great Depression: Social Change in Life Experience, University of Chicago Press, Chicago, 1974.

Elder, G. H.: The Life Course as Developmental Theory, Child Dev., 69, 1-12, 1998.

Esser, H.: Ist das Konzept der Assimilation überholt?, Geographische Revue, 5, 5-22, 2003.

Eurostat: Migration and Migrant Population Statistics, online aufrufbar: http://ec.europa.eu/eurostat/statistics-explained/ index.php/File:Immigration_by_citizenship,_2014_(\%C2\%B9) _YB16.png, letzter Zugriff: 20.10.2016.

Fechter, A.-M.: Gender, Empire, Global Capitalism: Colonial and Corporate Expatriate Wives, in: The New Expatriate. Postcolonial Approches to Mobile Professionals, Herausgeber: Fechter, A.-M. und Walsh, K., Routledge, Abingdon, 91-109, 2012.

Feijten, P. und van Ham, M.: Die Auswirkungen von Scheidung und Trennung auf die räumliche Mobilität in Großbritannien, Comparative Population Studies, 38, 433-464, 2013.

Findlay, A., McCollum, D., Coulter, R., und Gayle, V.: New Mobilities Across the Life Course: a Framework for Analysing Demographically Linked Drivers of Migration, Popul. Space Place, 21, 390-402, 2015.
Föbker, S., Pfaffenbach, C., Temme, D., und Weiss, G.. Hemmnis oder Hilfe - die Rolle der Familie bei der Eingliederung ausländischer Hochqualifizierter in den lokalen Alltag, in: Migration, Familie und Gesellschaft. Beiträge zu Theorie, Kultur und Politik, Herausgeber: Geisen, T., Studer, T., und Yildiz, E., Springer VS, Wiesbaden, 257-278, 2014.

Föbker, S., Imani, D., Nipper, J., Otto, M., und Pfaffenbach, C.: Translocal Life and Integration of Highly-skilled Migrants in Germany, Erdkunde, 70, 109-124, 2016.

Gramespacher, E., Funk, J., und Rothäusler, I. (Hrsg.): Dual Career Couples an Hochschulen. Zwischen Wissenschaft, Praxis und Politik, Budrich, Opladen, 2010.

Gupta, R., Banerjee, P., und Gaur, J.: Exploring the role of the spouse in expatriate failure: a grounded theory-based investigation of expatriate' spouse adjustment issues from India, Int. J. Hum. Resour. Man., 23, 3559-3577, 2012.

Hagan, J., MacMillan, R., und Wheaton, B.: New Kid in Town: Social Capital and the Life Course Effects of Family Migration on Children, Am. Sociol. Rev., 61, 368-385, 1996.

Hanganu, E. und Heß, B.: Die Blaue Karte EU in Deutschland. Kontext und Ergebnisse der BAMF-Befragung, Forschungsbericht 27, Bundesamt für Migration und Flüchtlinge, Nürnberg, 2016.

Hatfield, M. E.: Children moving "home"? Everyday experiences of return migration in highly skilled households, Childhood, 17, 243-257, 2010.

Hoinle, B. und Calba, S.: Editorial, Feministisches Geo-RundMail $62,2-4,2015$.

Holloway, S., Hubbard, P., Jöns, H., und Pimlott-Wilson, H.: Geographies of Education and the Significance of Children, Youth and Families, Prog. Hum. Geog., 34, 583-600, 2010.

Hutchins, T.: "They Told Us in a Curry Shop": Child-Adult Relations in the Context of Family Migration Decision-Making, J. Ethn. Migr. Stud., 37, 1219-1235, 2011.

James, A. und James, A. L.: Childhood: Toward a Theory of Continuity and Change, Ann. Am. Acad. Polit. Ss., 575, 25-37, 2001.

Jenks, C.: Constructing Childhood Sociologically, in: An Introduction to Childhood Studies, Herausgeber: Kehily, M. J., Open University Press, Maidenhead, 93-111, 2009.

Jurt, L. und Roulin, C.: „Es war ein Opfer, welches wir erbrachten ..." Perspektiven auf Migration in Familien, Gender, 1, 129144,2015

Kolb, H.: Internationale Mobilität von Hochqualifizierten - (k)ein Thema für die Migrationsforschung, in: Neue Zuwanderergruppen in Deutschland, Herausgeber: Swiaczny, F. und Haug, S., Bundesinstitut für Bevölkerungsforschung, Wiesbaden, 159174, 2006.

Krause-Nicolai, D.: Dual Career Couples im internationalen Einsatz. Implikationen für das internationale Personalmanagement, Lang, Frankfurt am Main, 2005.

Kupka, B. und Cathro, V.: Desperate housewives - social and professional isolation of German expatriated spouses, Int. J. Hum. Resour. Man., 18, 951-968, 2007.

Linehan, M.: Senior female international managers: empirical evidence from Western Europe, Int. J. Hum. Resour. Man., 13, 802814, 2002.

Millar, J. und Salt, J.: Portfolios of Mobility: the movement of expertise in transnational corporations in two sectors - aerospace and extractive industries, Global Netw., 8, 25-50, 2008. 
Müller-Bachmann, E.: The Family as a Unit: Experiences, Compromises and Negotiations in the Acculturation Process, in: Migration, Familie und Gesellschaft, Herausgeber: Geisen, T., Studer, T., und Yildiz, E., Springer VS, Wiesbaden, 113-130, 2014.

Mulder, C. H.: The Family Context and Residential Choice: A Challenge for New Research, Popul. Space Place, 13, 265-278, 2007.

NiLaoire, C., Carpena-Méndez, F., Tyrell, N., und White, A.: Introduction: Childhood and migration - mobilities, homes and belongings, Childhood, 17, 155-162, 2010.

ONS: A Century of Home Ownership and Renting in England and Wales, 2013, online aufrufbar: http: //webarchive.nationalarchives.gov.uk/20160105160709/http:

//www.ons.gov.uk/ons/rel/census/2011-census-analysis/ a-century-of-home-ownership-and-renting-in-england-and-wales/ short-story-on-housing.html (letzter Zugriff: 22.06.2017), 2013.

Portes, A. und Rumbaut, R.: Legacies, University of California Press, Oakland, 2001.

Purkayastha, B.: Skilled migration and cumulative disadvantage: the case of highly qualified Asian Indian immigrant women in the US, Geoforum, 36, 181-196, 2005.

Pusch, B.: Familiäre Orientierungen und Arbeitsmarktintegration von hochqualifizierten MigrantInnen in Deutschland, Kanada und der Türkei, in: Kulturelles Kapital in der Migration, Herausgeber: Nohl, A.-M., Schittenhelm, K., Schmidtke, O., und Weiß, A., VS Verlag, Wiesbaden, 285-300, 2010.

Pries, L.: Transnationalismus, Migration und Inkorporation. Herausforderungen an Raum- und Sozialwissenschaften, Geographische Revue, 5, 23-39, 2003.

Raghuram, P.: The difference that skills make: gender, family migration strategies and regulated labour markets, J. Ethn. Migr. Stud., 30, 303-321, 2004.
Roos, H.: In the Rhythm of the Global Market: Female Expatriates and Mobile Careers: A Case Study of Indian ICT Professionals on the Move, Gend Work Organ, 20, 147-157, 2013.

Ryan, L. und Sales, R.: Family Migration: The Role of Children and Education in Family Decision-Making Strategies of Polish Migrants in London, Int. Migr., 51, 90-103, 2013.

Salazar-Parreñas, R.: Children of Global Migration. Transnational Families and Gendered Woes, Stanford University Press, Stanford, 2005.

Sime, D. und Fox, R.: Migrant Children, Social Capital and Access to Services Post-Migration: Transitions, Negotiations and Complex Agencies, Children and Society, 29, 524-534, 2015.

Skelton, T.: Children's Geographies/Geographies of Children: Play, Work, Mobilities and Migration, Geography Compass, 3/4, 1430-1448, 2009.

Smith, D.: Geographies of long-distance family migration: Moving to a "spatial turn", Prog. Hum. Geog., 35, 652-668, 2011.

Vogel, D. und Rinke, B.: Sprache als der Schlüssel zur Integration? Theoretische Überlegungen und qualitativ-empirische Befunde zu Sprachfunktion im Integrationsprozess, in: Migration und städtischer Raum. Chancen und Risiken der Segregation und Integration, Herausgeber: Hillmann, F. und Windzio, M., Budrich, Opladen, 261-277, 2008.

Weiss, G., Otto, M., und Imani, D.: Die Bedeutung internationaler Schulen für Mobilitätsstrategien von Hochqualifizierten, Journal of International Mobility, 14, 165-183, 2014. 